\title{
Does the maternal vaginal microbiota play a role in seeding the microbiota of neonatal gut and nose?
}

\author{
O. Sakwinska ${ }^{1}$, F. Foata ${ }^{1}$, B. Berger ${ }^{1}$, H. Brüssow ${ }^{1}$, S. Combremont ${ }^{1}$, A. Mercenier ${ }^{1}$, S. Dogra ${ }^{2,3}$, S.-E. Soh ${ }^{2,4}$, \\ J.C.K. Yen ${ }^{5}$, G.Y.S. Heong ${ }^{6,7,8}$, Y.S. Lee ${ }^{2,3,9}$, F. Yap ${ }^{10}$, M.J. Meaney ${ }^{2,11}$, Y.-S. Chong ${ }^{2,8}$, K.M. Godfrey ${ }^{12,13}$ and $^{2}$ \\ J.D. Holbrook 2,13
}

${ }^{1}$ Nestlé Research Center, Vers-Chez-Les-Blanc, 1000 Lausanne, Switzerland; ${ }^{2}$ Singapore Institute for Clinical Sciences (SICS), Agency for Science and Technology Research (A*STAR), 30 Medical Drive, 117609 Singapore; ${ }^{3}$ Department of Paediatrics, Yong Loo Lin School of Medicine, National University of Singapore, 1E Kent Ridge Road, NUHS Tower Block, 119228 Singapore; ${ }^{4}$ Vishuo BioMedical Pte Ltd, 03-33/35A, Teletech Park, 2 O Science Park Road, Singapore; ${ }^{5}$ Department of Reproductive Medicine, KK Women's and Children's Hospital, 100 Bukit Timah Road, 229899 Singapore; ${ }^{6}$ Department of Maternal Fetal Medicine, KK Women's and Children's Hospital, 100 Bukit Timah Road, 229899 Singapore; ${ }^{7}$ Duke-NUS Medical School, 8 College Road, 169857 Singapore; ${ }^{8}$ Department of Obstetrics and Gynaecology, Yong Loo Lin School of Medicine, National University of Singapore, National University Health System, 1E Kent Ridge Road, 119228 Singapore; ${ }^{9}$ Division of Paediatric Endocrinology and Diabetes, Khoo Teck Puat - National University Children's Medical Institute, National University Health System, 1 E Kent Ridge Road, 119228 Singapore; ${ }^{10}$ Department of Paediatric Endocrinology, KK Women's and Children's Hospital, 100 Bukit Timah Road, 229899 Singapore; ${ }^{11}$ Ludmer Centre for Neuroinformatics and Mental Health, Douglas University Mental Health Institute, McGill University, 3755 Côte-Ste-Catherine Montreal, QC H3T $1 E 2$ Canada; ${ }^{12}$ MRC Lifecourse Epidemiology Unit, University of Southampton and University Hospital Southampton NHS Foundation Trust, Tremona Road, Southampton SO16 6YD, United Kingdom; ${ }^{13}$ NIHR Southampton Biomedical Research Centre, University of Southampton and University Hospital Southampton NHS Foundation Trust, MP 218 Tremona Road, SO16 6YD Southampton, United Kingdom; olga.sakwinska@rdls.nestle.com

Received: 23 May 2017 / Accepted: 12 September 2017

(c) 2017 Wageningen Academic Publishers

\section{OPEN ACCESS (c)(1)(2) RESEARCH ARTICLE}

\begin{abstract}
The acquisition and early maturation of infant microbiota is not well understood despite its likely influence on later health. We investigated the contribution of the maternal microbiota to the microbiota of infant gut and nose in the context of mode of delivery and feeding. Using $16 \mathrm{~S}$ rRNA sequencing and specific qPCR, we profiled microbiota of 42 mother-infant pairs from the GUSTO birth cohort, at body sites including maternal vagina, rectum and skin; and infant stool and nose. In our study, overlap between maternal vaginal microbiota and infant faecal microbiota was minimal, while the similarity between maternal rectal microbiota and infant microbiota was more pronounced. However, an infant's nasal and gut microbiota were no more similar to that of its own mother, than to that of unrelated mothers. These findings were independent of delivery mode. We conclude that the transfer of maternal vaginal microbes play a minor role in seeding infant stool microbiota. Transfer of maternal rectal microbiota could play a larger role in seeding infant stool microbiota, but approaches other than the generally used analyses of community similarity measures are likely to be needed to quantify bacterial transmission. We confirmed the clear difference between microbiota of infants born by Caesarean section compared to vaginally delivered infants and the impact of feeding mode on infant gut microbiota. Only vaginally delivered, fully breastfed infants had gut microbiota dominated by Bifidobacteria. Our data suggest that reduced transfer of maternal vaginal microbial is not the main mechanism underlying the differential infant microbiota composition associated with Caesarean delivery. The sources of a large proportion of infant microbiota could not be identified in maternal microbiota, and the sources of seeding of infant gut and nasal microbiota remain to be elucidated.
\end{abstract}

Keywords: microbiota transfer, delivery mode, perinatal microbiota 


\section{Introduction}

The microbiota of human body sites is thought to exert profound influence on the physiology, health and disease of its human host. At the moment of birth, a healthy new-born transits from a generally sterile environment to extensive microbial exposure. The gut microbiota has received more attention than other body sites because of its sheer size (Huttenhower et al., 2012) and demonstrated impact on its human host. Some of the clearest evidence concerns necrotizing enterocolitis (NEC) in premature infants, where gut microbiota composition is highly predictive of disease (Sim et al., 2015; Warner et al., 2016). Emerging evidence suggests that the differential acquisition and maturation of gut microbiota in healthy infants may have consequences for health later in life (Arrieta et al., 2015; Bisgaard et al., 2011; Dogra et al., 2015; Korpela et al., 2016). The microbiome of the upper respiratory tract, which contains the second most abundant bacterial population in the human body, has been less explored. In infants the respiratory microbiome may be particularly important, as the upper respiratory tract is the site of many infections, which are one of the main health concerns at this life stage. The respiratory tract microbiome is increasingly considered to play an important role as modulator of respiratory infections as well as other respiratory disorders, such as wheezing and asthma (Bisgaard et al., 2007; Teo et al., 2015; Van den Bergh et al., 2012).

The infant microbiome derives from the immediate environment and the colonising microbes must be able to persist on or in the body. Thus members of immediate family are thought to be the most likely source of the microbes that colonise neonates (Bäckhed et al., 2015; Dominguez-Bello et al., 2010). Other sources remain poorly understood. After the initial microbial inoculum has been acquired, selection will occur within the infant body. While some infant body niches might provide relatively similar growth conditions for the microbes transmitted by adults (putatively the upper respiratory tract), some differ radically. For instance, the bacteria in the infant gut receive a very specific food substrate in form of breast milk or infant formula, and infant gut microbiota is radically different to adults' (Yatsunenko et al., 2012).

The strong influence of delivery and feeding modes on infant gut microbiota composition has been demonstrated (Adlerberth and Wold, 2009; Azad et al., 2015; Bäckhed et al., 2015; Bokulich et al., 2016). The impact of the delivery mode is the most pronounced in the first days and weeks of life (Dogra et al., 2015; Stokholm et al., 2016). In the very first days and weeks of an infant's life, his or her gut microbiota often undergoes substantial fluctuations in composition. Transient presence of bacteria that are unable to propagate and permanently colonise may be thought of little relevance. However, there is mounting evidence that microbe-host interactions are crucial for the processes of immune maturation taking place soon after birth (Gensollen et al., 2016). It can thus be hypothesised that patterns of even transient early microbial contact may have far reaching physiological consequences, not necessarily evident as a lasting influence on microbiota composition.

Here we characterised the microbiota of 42 mother-infant pairs from the Growing Up in Singapore Towards healthy Outcomes (GUSTO) birth cohort (Soh et al., 2014) by $16 \mathrm{~S}$ rRNA gene sequencing. Microbiota from the vagina, rectum and skin of mothers as well as from infant stool and nose were investigated. The impacts of delivery mode and feeding mode on the first stages of colonisation and establishment of gut and nasal microbiota are described, along with the role of maternal microbiota in seeding of infant gut and nasal microbiota.

\section{Material and methods}

\section{Subjects}

From the GUSTO birth cohort (Soh et al., 2014), we selected a sub-group of 42 mother-infant pairs. All consecutive participants recruited between July and September 2010 were asked to participate in this part of the study. This study was approved by both the National Healthcare Group Domain Specific Review Board (reference no. D/09/021) and the Sing Health Centralized Institutional Review Board (reference no. 2009/280/D). All of the participating mothers provided written informed consent. Subject characteristics are given in Table 1. The mothers and infants in this study were healthy, and of normal gestational age (Table 1). Infants and mothers reported no medical problems during the study period except one case of duodenal atresia that was surgically repaired and one case of transient tachypnea that needed no treatment. We considered that infants with these conditions represent usual variability of the population and should not be excluded from the study. Mother-infant pairs spent on average 2.1 days in the hospital from the delivery to the discharge and it is usual for infants to be roomed with their mothers, although we did not record if there were occasional periods of separation. At $1 \mathrm{~min}$, the average Apgar score was 8.7, and at $5 \mathrm{~min}$ it was 9 for all infants The rate of Caesarean section of $19 \%$ is lower than typical rates for Singapore, because complicated deliveries were underrepresented due to logistic reasons. All cases of Caesarean section were elective (unlaboured). Disinfection of the maternal perineum by external swabbing with $2 \%$ chlorhexidine gluconate solution just before vaginal delivery was routinely practiced. The maternal vaginal and rectal swabs were taken before disinfection was done. None of the infants received antibiotics apart from the infant who required surgery for duodenal atresia. Otherwise, the mother-infant pairs in this study were similar to the larger GUSTO cohort. Most infants were mix-fed with 
Table 1. Basic characteristics of mothers and infants included in this study and comparison with the entire Gusto cohort. ${ }^{1}$

\begin{tabular}{|c|c|c|c|c|c|c|c|c|}
\hline \multirow[b]{2}{*}{ Parameter } & \multicolumn{4}{|c|}{42 mother-infant pairs included in this study } & \multicolumn{4}{|c|}{ All data available for entire GUSTO cohort } \\
\hline & Mean & SD & Min & Max & Mean & SD & Min & Max \\
\hline Maternal age (years) & 30.6 & 5.07 & 19 & 40 & 30.6 & 5.17 & 18 & 46 \\
\hline Gestation duration (weeks) & 38.93 & 1.13 & 36 & 41 & 38.29 & 1.6 & 25 & 41 \\
\hline Infant weight at birth $(\mathrm{kg})$ & 3.2 & 0.43 & 2.4 & 4.3 & 3.08 & 0.46 & 0.78 & 5.43 \\
\hline \multirow[t]{2}{*}{ Infant length at birth $(\mathrm{cm})$} & 49.13 & 1.94 & 45 & 53 & 48.53 & 2.34 & 34 & 57 \\
\hline & $\mathrm{n}(\%)$ & & & & $\mathrm{n}(\%)$ & & & \\
\hline Vaginal delivery & $32(76)$ & & & & $753(64)$ & & & \\
\hline Vaginal delivery with vacuum & $2(5)$ & & & & $44(4)$ & & & \\
\hline Vaginal delivery with forceps & 0 & & & & $22(2)$ & & & \\
\hline Emergency Caesarean section & $6(14)$ & & & & $236(20)$ & & & \\
\hline Elective Caesarean section & $2(5)$ & & & & $114(10)$ & & & \\
\hline Exclusive breastfeeding until week 1 & $9(22)$ & & & & $224(23)$ & & & \\
\hline Exclusive breastfeeding until week 3 & $10(24)$ & & & & $251(25)$ & & & \\
\hline Antibiotics exposure $\leq 31$ days before birth & $15(36)$ & & & & $436(37)$ & & & \\
\hline
\end{tabular}

breastfeeding supplemented with formula. Participants in the study were not provided with advice on the choice of infant milk formula. Consequently, the infants were fed a large range of different formulas, including brands containing probiotics (around a third of infants received them). Samples were collected from the rectum, vagina and breast skin of the mother at delivery. In infants, nasal samples were collected at birth and at 3 weeks old, while stool samples were collected at 3 days old and 3 weeks old. Apart from stool samples, the collection was performed with transport system swabs (Copan, Brescia, Italy). The samples were frozen immediately after collection.

\section{S rRNA sequencing and qPCR}

Overall, 239 samples were collected from 42 motherinfant pairs. A complete set of samples was not available for all subjects because of issues with logistics and subjects dropping out of the study. Processing of the biological samples, and generation of $16 \mathrm{~S}$ rRNA sequencing data was as described in (Bokulich et al., 2013; Dogra et al., 2015). Briefly, the variable regions V4-V5-V6 (V456) of the $16 \mathrm{~S}$ rRNA gene were used to characterise the microbiota by the barcoded-primer approach to multiplex pyrosequencing. Raw sequence data were analysed using Mothur v.1.33.0 (Schloss et al., 2009) and QIIME v.1.8 (Caporaso et al., 2010) software packages. Pyrosequencing reads were denoised with the Mothur implementation of PyroNoise (Quince et al., 2009) according to the 454 SOP described in (Schloss et al., 2009). Chimeras were identified using usearch61 in QIIME (Edgar et al., 2011). The sequences were then trimmed as described in the Mothur 454 SOP in order to keep sequences overlapping the same $16 \mathrm{~S}$ region. Subsequent analytical steps were performed in QIIME. Operational taxonomic units (OTUs) de novo picking at 97\% identity was performed using uclust (Edgar, 2010). Taxonomy assignment of OTU representative sequences used the RDP Classifier with confidence threshold of 0.6 (Wang et al., 2007) on the Greengenes reference database v.13.8 (McDonald et al., 2012). After quality filtering (Bokulich et al., 2013), similarities between all samples were computed as Binary Jaccard (for similarity in community membership) or Bray Curtis (for similarity in community structure) distances using OTUs (the lowest discriminant units with this technology). Diversity analyses were calculated in QIIME on data rarefied at 200 reads per sample. This led to exclusion of three samples with lower number of sequencing reads.

Microbiota composition could be evaluated in 168 samples, $70 \%$ of the total. This led to the exclusion of two motherinfant pairs from any analyses. A total of 174,827 sequencing reads were obtained and 436 OTUs were identified. Given the diversity of the sample set, a relatively low number of OTU indicated that majority of them were genuine. Rectal samples were shown to closely approximate the stool samples (David et al., 2015). This was the case in our study, where typical adult stool taxa were identified including Bacteroides, Prevotella, Erysipelotrichaceae, 
Lachnospiraceae, Clostridiaceae, Ruminococceae (including Faecalibacterium), Verrucomicrobiaceae (including Akkermansia). Moreover, out of 215 OTUs identified in rectal samples, 95 were unique to rectal samples and not detected in maternal vaginal or skin samples (Supplementary Table S1). The success rate of obtaining interpretable sequencing data varied among different niches with the greatest success for maternal rectal and vaginal, and infant stool samples, reduced success for infant nasal samples at 3 weeks old, and limited success for maternal breast skin and infant nasal samples at birth (Supplementary Table S2). The most likely explanation for this variability is very differential abundance of bacterial DNA (Figure 1A).

The impact of feeding mode on nasal microbiota could not be evaluated because there were only four samples from exclusively breastfed infants where microbiota could be assessed at day 3. Similarly, the impact of delivery mode on nasal microbiota at birth could not be evaluated because only one sample from Caesarean section delivered infant was available (Supplementary Table S2). To determine whether the samples differed in overall microbiota composition, permutation-based analysis of variance was applied (permanova). The distribution of beta diversity measures of bacterial community were performed with Mann-Whitney or Kruskal-Wallis test. Unadjusted $P$-values are reported because specific hypotheses on pairwise comparisons were tested. Paired tests were performed with Wilcoxon's matched pair test. Shannon diversity indexes were compared with Student t-test.

Quantitative PCR on total bacteria, Staphylococcus aureus and total lactobacilli were performed according to published methods (Alarcón et al., 2006; Nadkarni et al., 2002; Penders et al., 2006). The bacterial loads were compared with Mann-Whitney test. Accession number to sequencing data deposited in the GenBank Short Read Archive is SRP081351.

\section{Results}

\section{Microbiota abundance and composition differed between sample types.}

The microbiota sampled from different body sites at different time points differed both in bacterial abundance (Figure 1A) and composition (Figures 1B-C). Despite high variability among individuals at the same site, the difference in composition between sample sites was highly significant (Figure 1B, permanova, $P<0.001$ ). The body sites were strongly differentiated (Figure 1C, Mann Whitney test on Bay Curtis distances, $P<0.001)$. In pairwise comparison, the only sites which could not be differentiated based on Bray-Curtis distances analyses, reflecting similarities in their community structures, were maternal breast skin and infant nasal samples at week 3 , and infant stool samples collected at day 3 and week 3 (Figure 1C, Mann-Whitney test on Bay Curtis distances, $P=0.72$ ).

The maternal vaginal microbiota was dominated by lactobacilli, while the rectal microbiota was more diverse, with Prevotella, Bacteroides and Escherichia among the dominant genera. Breast skin microbiota mostly consisted of Staphylococcus and Corynebacterium (Figure 1B). For the maternal samples, total bacterial load (expressed as genome equivalent per $\mathrm{ml}$ sample) was highest in the rectal and vaginal samples, and lowest in the breast skin samples (Figure 1A).

Infant stool microbiota at day 3 consisted mainly of Bifidobacterium, Streptococcus, Klebsiella and Escherichia, with dominance of Bifidobacterium established by week 3 (Figure 1B). Between day 3 and week 3, there was a small but significant change in stool microbiota composition (Figure $1 \mathrm{~B}$, permanova, $P=0.028$ ), however, the dissimilarity in terms of Bray Curtis distances distributions was not significant (Supplementary Table S3, Mann-Whitney, $P=0.17)$. The high bacterial load in stool samples at 3 days old remained unchanged at 3 week old (Mann-Whitney test including all samples, $P=0.64$, Wilcoxon test pairing all available samples within individual, $P=0.35$ ), (Figure 1A).

Infant nasal microbiota underwent a large change in composition between birth and week 3 of life (Figure 1B, permanova, $P<0.001)$. This was reflected in significant dissimilarity of community structure distributions (Figure $1 C$, Mann Whitney test on Bray Curtis distances, $P<0.001)$. Diverse at birth, in 3 weeks old infants the nasal microbiota became dominated by Staphylococcus and Corynebacterium, while Streptococcus and Pseudoxanthomonas abundance decreased (Figure 1C). Low bacterial load in nasal samples at birth (Figure 1A) increased 10-fold at 3 weeks (MannWhitney test including all samples, $P<0.0001$, Wilcoxon test pairing all available samples within individual, $P<0.001$ ). The reductions in within-sample diversity (Supplementary Figure S1, $P=0.007$, t-test) was accompanied by a reduction in between-sample diversity, in other words the individual samples resembled each other more with time (Supplementary Figure S2, $P<0.001$, Mann-Whitney test).

The potentially pathogenic $S$. aureus could not be detected in the investigated maternal body sites when 35 rectal samples, 32 vaginal samples and 29 of breast skin samples were assessed by qPCR. The prevalence of $S$. aureus in nasal samples of the infants increased from $6 / 40$ (15\%) at birth to $22 / 32(69 \%)$ at 3 weeks old. 


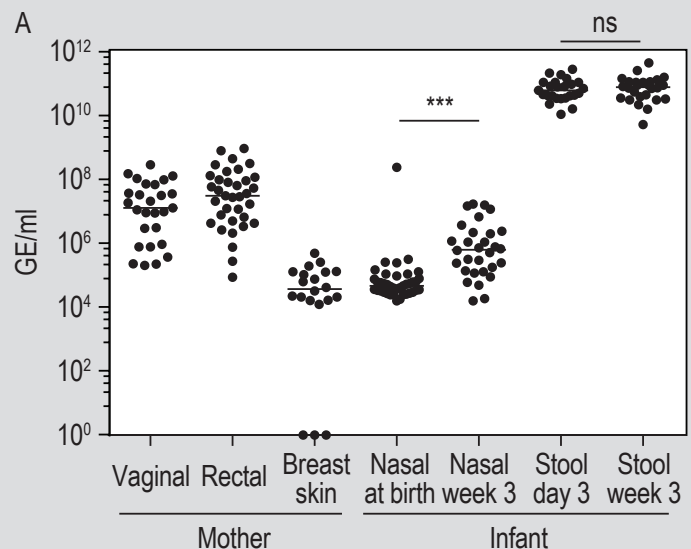
(27)
(35)
(20)
(39)
(30)
(25)
(26)
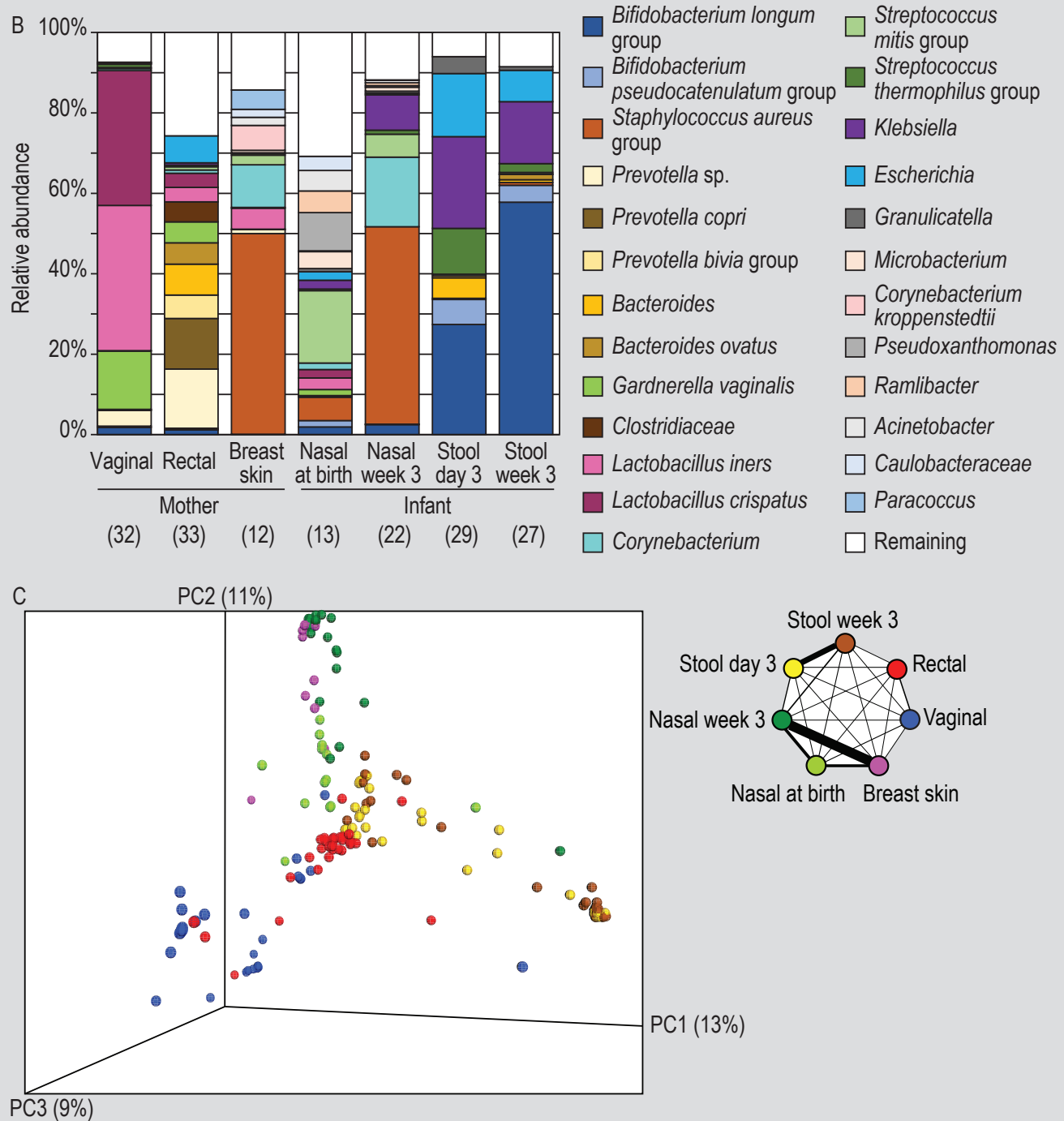

Figure 1. Microbiota characteristics from different body sites of mothers and infants. (A) Total bacterial load. The line represents the median; ns $=$ non-significant, $P=0.64 ;{ }^{* * *}, P<0.0001$, Mann-Whitney test. Wilcoxon's matched within individuals pair test: nasal, $\mathrm{n}=28, P<0.001$; stool, $\mathrm{n}=20, P=0.349$. The number of samples available for evaluation is given in brackets. (B) Relative abundance of bacterial taxa classified down to species level. Reads representing $<3 \%$ of total were pooled and are labelled 'Remaining'. (C) Principal coordinate (PCoA) analysis of Bray Curtis distances among samples. Inset: the thickness of the lines represents the similarity between the niches (line weight is inversely proportional to pairwise Bray Curtis distances). The distances and corresponding $P$-values are listed in Supplementary Table S3. 


\section{Association of delivery mode and feeding with infant gut microbiota composition}

Both delivery mode and feeding mode were associated with differences in the overall composition of infant stool microbiota at day 3 (permanova, delivery $P=0.003$; feeding $P<0.001$, Mann Whitney test on Bray Curtis distances distributions, delivery $P<0.001$; feeding $P=0.002$ ).

We compared the stool microbiota of the eight vaginally delivered and exclusively breastfed (V-B) infants with the ten who were vaginally delivered and mix-fed (V-M, Figure 2A). Mixed feeding resulted in decreased Bifidobacterium and increased Enterobacteriaceae, Klebsiella, Escherichia., and Streptococcus contribution while the proportion of Bacteroides remained unchanged.

Comparison of ten V-M and eight Caesarean-section delivered and mix-fed (C-M) infants allowed us to assess the impact of Caesarean section among those who were mix-fed. C-M infants showed lower Bifidobacterium levels compared to V-M, larger proportions of Klebsiella, and complete lack of Bacteroides. In this dataset, none of the infants delivered by Caesarean section were exclusively breastfed (Table 1), preventing comparison of breastfeeding with mixed feeding within the Caesarean section delivered group. Also, our dataset contained just two infants that were exclusively formula-fed so we could not perform comparisons to a formula fed only group. There were no significant differences in gestational age and infant birth length and weight among the V-M, V-B and C-M groups (Supplementary Table S4). Despite considerable differences in composition, the delivery and feeding modes had no significant impact on bacterial load in stool at Day 3 (Figure 2B).

\section{Similarity between infant and maternal microbiota by mode of delivery}

There was very little resemblance of the initial infant microbiota at any site to the microbiota of any of the maternal body sites (Figure 1B and 1C). The distances were close to 1, which represents the largest possible dissimilarity. Similarity of infant stool microbiota to maternal rectal microbiota was slightly greater than the similarity to maternal skin or vaginal microbiota (Supplementary Table S3). The magnitude of changes in similarity between maternal and infant microbiota over the study period was small, with the exception of increase of similarity between microbiota of maternal skin at delivery and infant nose collected at birth and at week 3 (Supplementary Table S5). distance) between maternal rectum and infant stool at day 3 , compared to vaginal delivery (Figure 3A, Supplementary Table S6). There was no effect of delivery mode on similarity to vaginal microbiota (Figure 3A, Supplementary Table S6). On the other hand, there was a trend for greater similarity between the microbiota of maternal skin and infant stool for Caesarean section delivered infants compared to vaginally delivered infants (Figure 3A, Supplementary Table S6).

At week 3, the resemblance in community membership between infant stool and maternal rectal microbiota was no longer impacted by the mode of delivery, as the distance between maternal rectal samples and the stools of Caesarean section delivered infants had decreased (compare Figures 3A vs 3B, Supplementary Table S5). There was still no significant difference by delivery mode in the distances between the microbiota of week 3 infant stool samples and maternal vagina. Also at week 3 , the shared membership between infant stool microbiota and maternal skin microbiota became significantly greater in Caesarean section delivered infants compared to vaginally delivered infants (Figure 3B, Supplementary Table S7).

Only one nasal sample was available for Caesarean section delivered infants at birth, so data is not shown. At week 3 , the resemblance between maternal rectal and skin and nasal microbiota did not significantly differ according to the delivery mode (Figure 3C, Supplementary Table S8). In contrast, the similarity to maternal vaginal microbiota was significantly greater for vaginally delivered infants compared to Caesarean section delivered infants (Figure 3C, Supplementary Table S8).

\section{Similarity between infant and maternal microbiota within mother-infant pairs is not greater than across unrelated mothers and infants}

It would be tempting to postulate that the observed pattern of decreased resemblance between rectal and vaginal maternal microbiota to infant stool and nasal microbiota following Caesarean delivery, was the result of reduced microbiota transfer from the mother.

However, the infant stool microbiota did not share more community members with the microbiota of their own mothers than with the microbiota of unrelated mothers at any of the maternal sites (Figure 4A-D, Supplementary Tables S9-S12). This was true for all considered time points and whether vaginally and Caesarean-section delivered infants were considered separately or both groups were pooled (Supplementary Tables S9-S12).
Nevertheless, the similarities in community membership between microbiotas of maternal sites and of infant stool were associated with delivery mode. Caesarean section was associated with a reduction of shared microbiota (i.e. greater 
A $P=0.001$

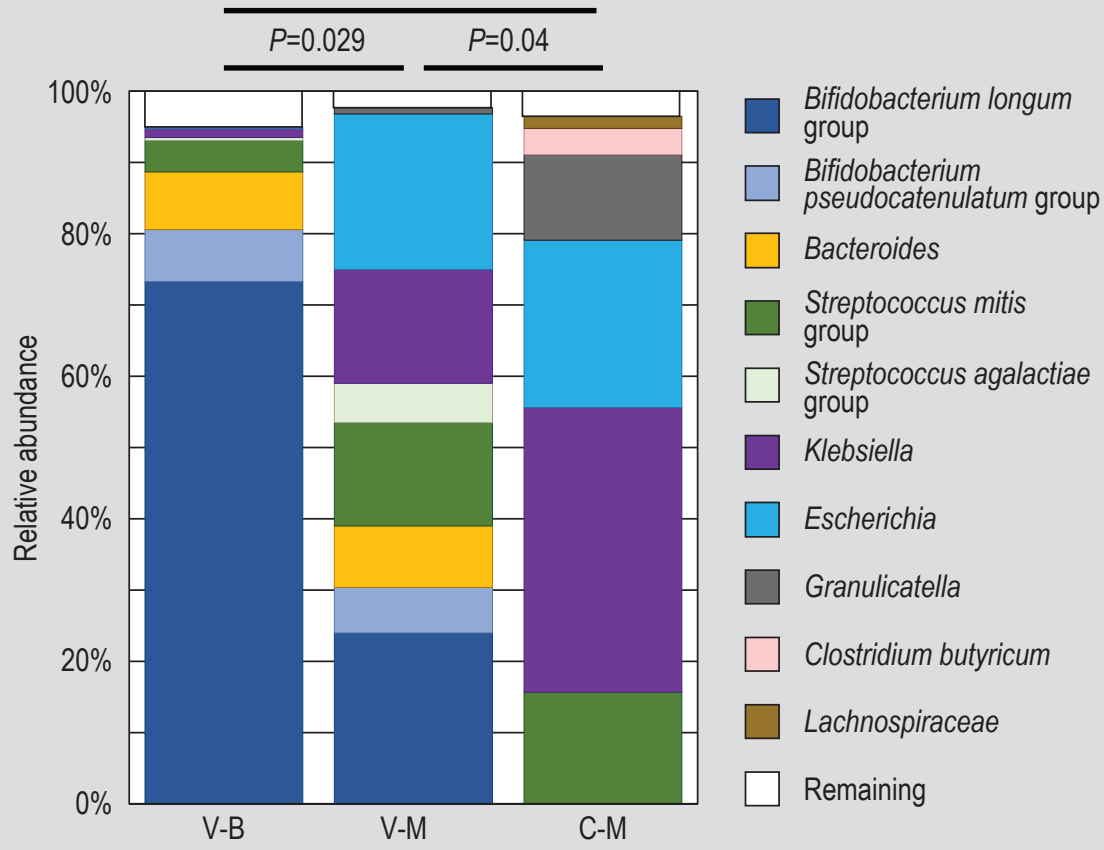

B

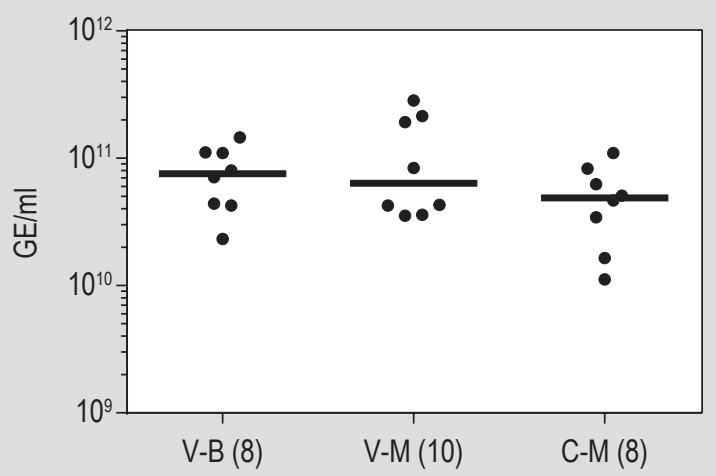

Figure 2. Association of the delivery mode and feeding mode on infant stool microbiota at day 3. (A) Microbiota composition in infant stool samples at day 3 expressed as relative abundance of bacterial taxa classified down to species level. Reads representing $<3 \%$ of total were pooled and are labelled 'Remaining'. $P$-values correspond to permanova. V-B denotes vaginally delivered and exclusively breastfed infants, V-M vaginally delivered and mix fed, and C-M, C-section delivered and mix fed, respectively. The number of samples available for evaluation is given in brackets. (B) Total bacterial load. The lines represent medians.

\section{Minor proportion of infant gut microbiota is shared with maternal vaginal microbiota}

To further elucidate the role of maternal microbiota as the source of microbial inoculum for the infant, we have calculated the proportions of OTUs detected in the infant stool samples that were shared with the microbiota of their own mothers. We limited this analysis to maternal rectal and vaginal samples and excluded breast skin samples because of low bacterial abundance resulting in low sequencing success for these samples (Supplementary Table S2) as well as overall high dissimilarity of microbiota of breast skin samples and infant stool samples (Supplementary Table S3).

More than $50 \%$ of OTUs identified in all the infant stool samples at day 3 were not observed in either rectal or vaginal maternal microbiota (Figure 5). However, there was a large degree of inter-individual variation: for one infant all the OTUs in its day 3 gut microbiota were also found in the maternal rectum, vagina or both; and for three infants none of the OTUs in their day 3 gut microbiota were found in the maternal samples. There were no significant differences in degree of shared microbiota between VD 
A

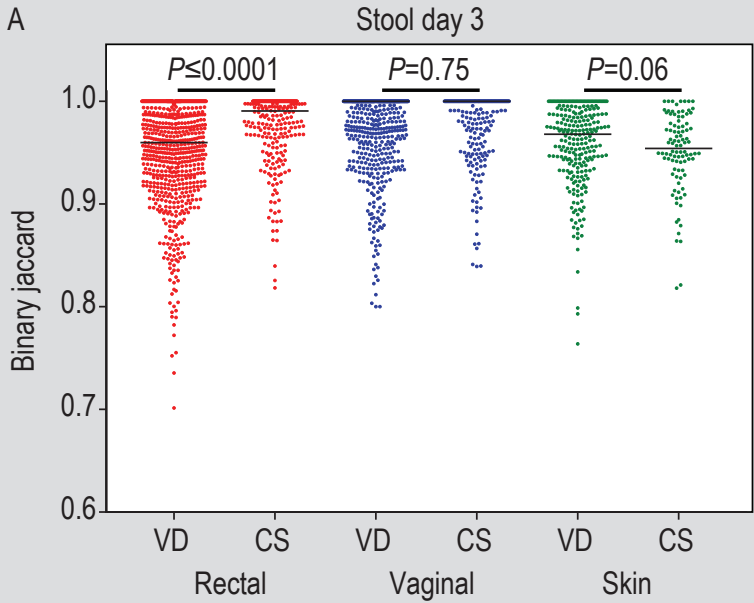

C

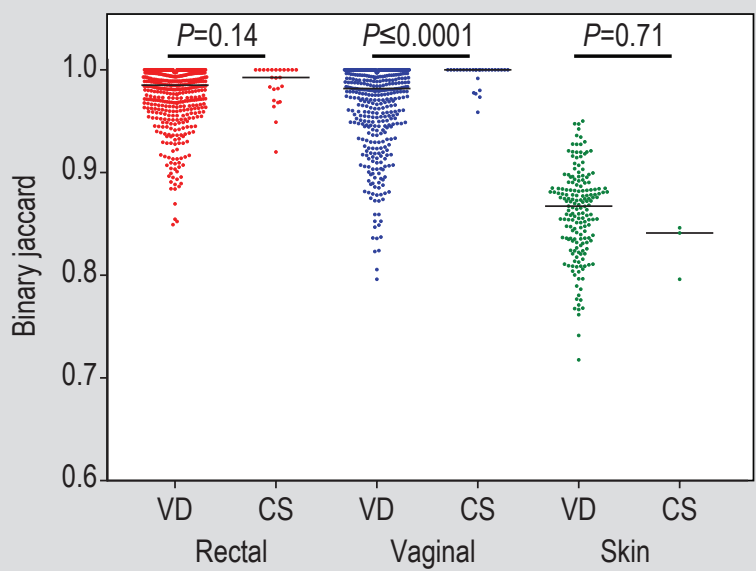

B

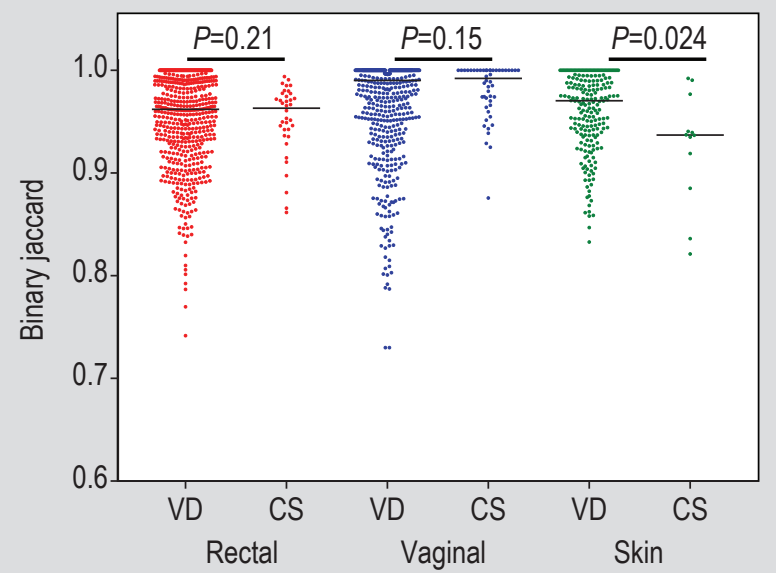

Figure 3. Microbial community distances between microbiota in maternal samples taken at delivery and infant samples. Y-axis, values are between $0-1$ with 1 representing maximum possible distance and 0 representing exactly the same microbiota content. Infant stool samples were taken at day 3 (A); and at week 3 (B); and infant nasal sample at week 3 (C) for infants delivered vaginally (VD) and by Caesarean section (CS). Thick black lines represent medians. $P$-values correspond to Kruskal-Wallis tests for differences between distances for VD or CS infants with unadjusted $P$-values. The details of statistical tests are in Supplementary Tables S6-S8.

and CS infants (MannWhitney test on proportion of shared OTUs, ns). The degree of overlap between infant and mother microbiota was larger for the maternal rectal than for vaginal microbiota (Mann-Whitney test, $P=0.004$ for CS and VD pooled; $P=0.06$ for VD; $P=0.15$ for CS).

Our analysis might have been restricted by limited sequencing depth and consequent limited ability to detect low-abundance taxa. We therefore supplemented our approach by more detailed quantitative analysis of lactobacilli which were the most dominant taxon in maternal vaginal samples. Quantification of total lactobacilli by qPCR in infant stool samples collected at day 3 revealed absence of lactobacilli. A minor proportion of nasal samples at birth contained low numbers of lactobacilli, while they were present in most vaginal samples in large numbers (Supplementary Figure S3).

\section{Discussion}

In agreement with earlier studies, we observed that microbiota residing in different body sites were highly differentiated (Chu et al., 2017; Huttenhower et al., 2012). This differentiation was already clear in 3 day old infants and very pronounced in 3 week old infants. Such strong differentiation suggests that the physiology and nutrients of the niche encountered by bacteria have decisive effects on microbiota composition. Counter-intuitive to the concept of maternal vaginal seeding of neonatal microbiota, initial infant faecal and nasal microbiota was highly dissimilar to microbiota at all three sampled maternal sites. Indeed, dissimilarity to 3 day old microbiota was greatest for maternal vaginal microbiota. However, at week 3 , the infant nasal microbiota and maternal breast skin microbiota converged to become the most similar pair among all sample types. We suggest that this convergent process 


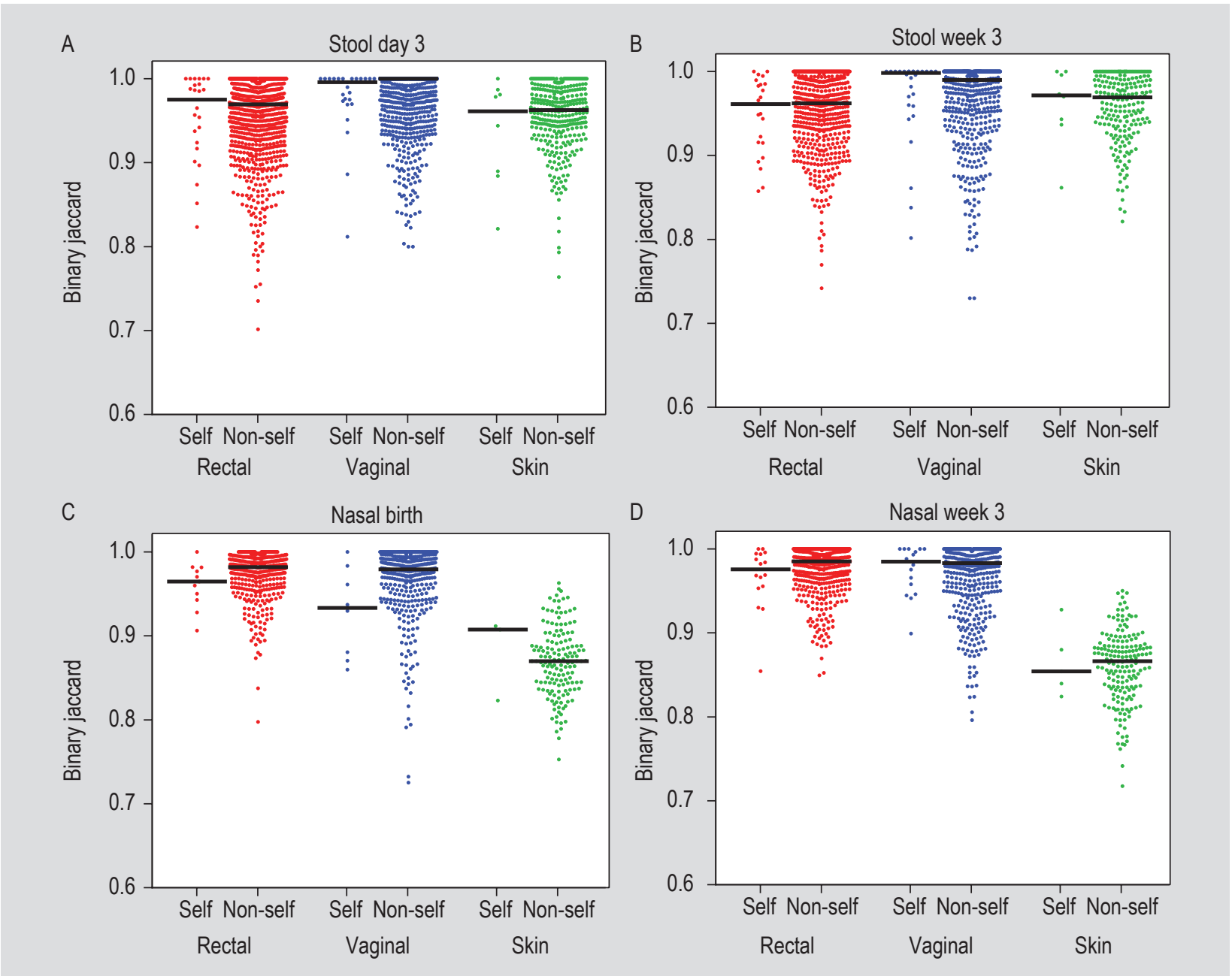

Figure 4. Microbial community distances between microbiota of infant samples to microbiota of their own mothers (Self) and to the microbiota of unrelated mothers (Non-Self). Y-axis, values are between 0-1 with 1 representing maximum possible distance and 0 representing exactly the same microbiota content. Maternal rectal, vaginal and breast skin samples were taken at delivery and infant samples were stool collected at day $3(A)$, stool collected at week 3 (B), nasal collected at birth (C) and at week 3 (D). Thick black lines represent medians. None of the comparisons between Self and Non-Self was significant according to KruskalWallis test with unadjusted $p$ values. The details of statistical tests are in Supplementary Tables S9-S12.

is driven by similar environmental conditions at the two body sites. Increase in bacterial load and decrease in within sample diversity between birth and week 3 may reflect quick elimination of most taxa unable to survive in the nose.

The impact of delivery mode and feeding mode on infant faecal microbiota were unambiguous, and consistent with earlier studies (Adlerberth and Wold, 2009; Bäckhed et al., 2015; Bokulich et al., 2016; Madan et al., 2016). The differences between vaginally delivered and Caesareansection delivered infants are apparent in early days and weeks and diminish with time (Dogra et al., 2015; Stokholm et al., 2016), which may explain the relatively minor effects reported in some studies (Chu et al., 2017). Vaginally delivered, breastfed infants had microbiota dominated by bifidobacteria. Compared to exclusive breastfeeding, partial formula feeding led to an increase in Enterobacteriaceae and streptococci at the expense of bifidobacteria, as early as day 3. Consistent with earlier studies (Azad et al., 2013; Bäckhed et al., 2015; Madan et al., 2016), Caesarean-section was associated with strong reduction of Bacteroides. In this sample set, none of the infants delivered by Caesarean section were exclusively breastfed, which likely reflects clinical reality in this population; also elsewhere Caesarean section is associated with lower rates of breastfeeding, e.g. (Prior et al., 2012). All of the Caesarean section deliveries in this study were performed before the onset of labour, so there was no exposure of the neonates to the vaginal microbiome (Prior et al., 2012).

It has been suggested that distinct exposure to the maternal microbiome experienced in vaginal and Caesarean section deliveries drives the effect of the delivery mode on neonatal microbiomes. If the mother provides the seed for microbes 


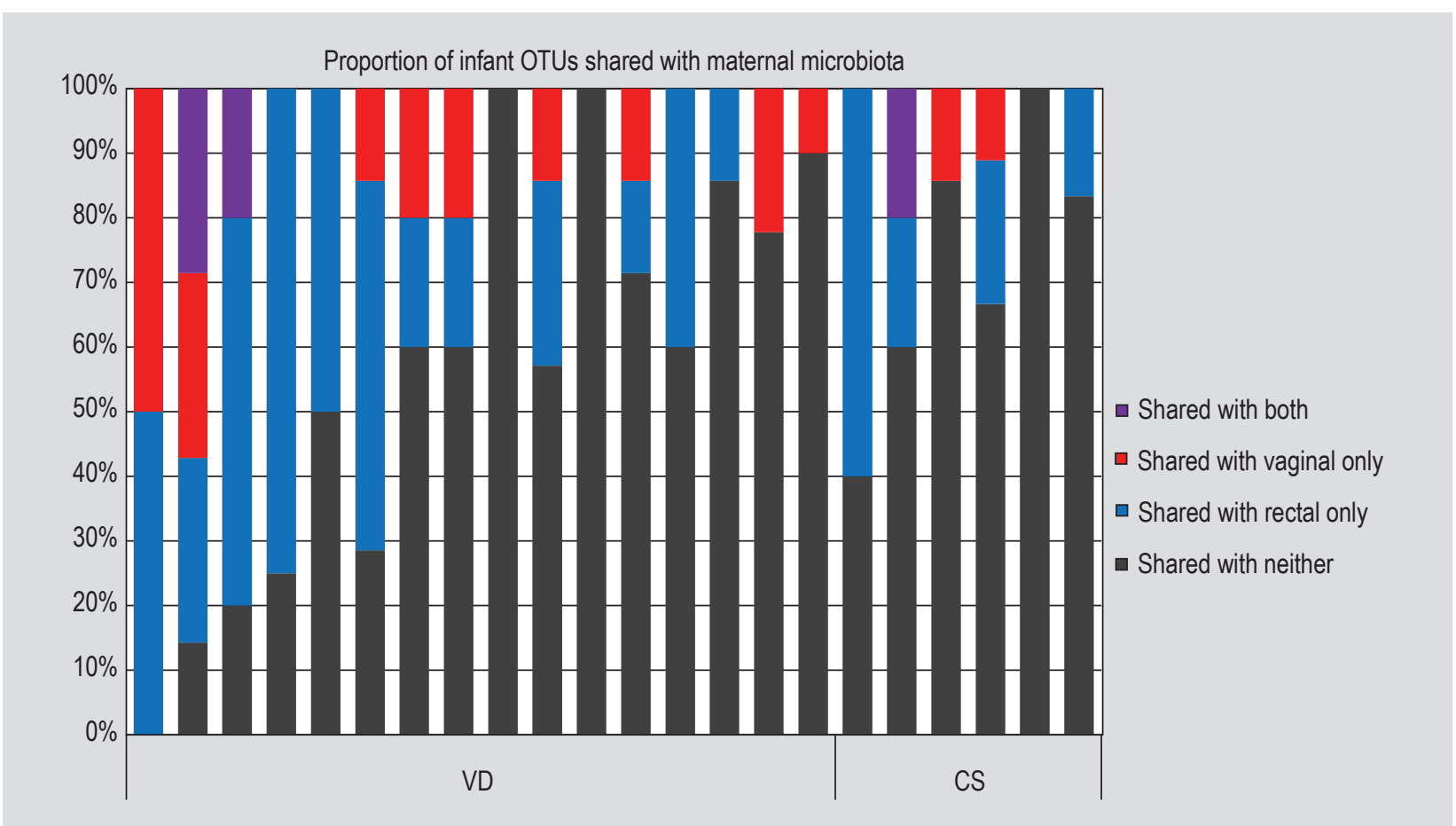

Figure 5. Proportion of infant operational taxonomic units (OTUs) shared between infant stool microbiota at day 3 and maternal microbiota. Only pairs with available three samples were analysed. VD denotes vaginally delivered and CS denotes Caesarean section delivered infants, respectively.

colonising the neonate, it follows that mother-infant pairs should display greater resemblance of infant microbiota in comparison with unrelated mothers (Bokulich et al., 2016; Dominguez-Bello et al., 2010). Greater resemblance to microbiota of perineal sites should be evident for vaginally delivered infants, while the similarity with skin microbiota should be greater after Caesarean section. Our study did not find evidence to support this hypothesis: infant microbiota did not resemble own mother's microbiota more than it did microbiota of unrelated mothers, at any site or time point. When the analysis was limited to vaginally delivered infants, no greater resemblance to own mother's microbiota was revealed than to unrelated mothers.

In particular, the similarity between maternal vaginal and infant stool microbiota sampled at day 3 of life was minimal. The fraction of microbiota shared was small even at OTU level, and these results were corroborated by qPCR data. We found no support for the role of maternal vaginal microbiota in the seeding of the majority of the infant gut and nasal microbiota. Dominguez-Bello and colleagues (2010) demonstrated more similarity of the vaginally delivered infant skin, oral and nasal microbiota to the vaginal microbiota of their own mothers compared to that of other mothers. However, in their study most of the sampling was performed minutes after the delivery and the maintenance of the similarity over the next hours, was not described. It is credible that the very transient passage of vaginal microbes through infant gut (or presence at low level as in the concept of keystone species (Faust and Raes, 2012)) leads to physiological changes and or triggers host response. Yet, transient passage, however consequential, should not be confused with seeding infant gut colonisation. Chu and colleagues (Chu et al., 2017) in a recent study of stool microbiota in 52 infants from the USA, found little difference in similarity to maternal vaginal OTUs between the infant stool microbiome sampled at six weeks relative to delivery mode. In a proof of concept study of 7 infants, Dominguez-Bello et al. (2016) showed that exposure of Caesarean section delivered infants to maternal vaginal microbiota resulted in infant skin microbiota that resembled that of vaginally delivered infants. However, exposure to vaginal microbiota had no impact on the anal microbiota of the infants (their stool microbiota was not investigated). These findings are consistent with minimal impact of maternal vaginal microbiota on infant gut microbiota and potential impact on infant skin microbiota; however, the clinical significance of the infant skin microbiome remains to be explored.

Seeding from maternal intestinal tract is the expected source of infant microbiota. In our study, the similarity between infant stool microbiota to maternal rectal microbiota was more pronounced than infant stool to maternal vaginal microbiota. However, the similarity within mother-infant pairs was not greater than between unrelated pairs, and this was irrespective of the delivery mode. Comparable results were obtained when the transfer from maternal stool 
and rectal microbiota to infant was assessed in a cohort of 43 infants and their mothers using 16S data (Bokulich et al., 2016). Although rectal microbiota of mother-infant pairs did resemble each other more than those of unrelated pairs, this similarity appeared only after 4 to 6 months after birth. Its absence in the first 6 months and increased similarity henceforth is more consistent with the fact that infants increasingly share maternal diet from 6 months onwards rather than transfer of gut microbiota at birth. Contrary to expectations, the resemblance in community membership between infant and mother stool microbiota for unrelated mothers was greater than resemblance to the microbiota of own mother. This is surprising, because the presence of identical strains of bifidobacteria strains in both maternal and infant stool was shown using culture and typing methods (Jost et al., 2013; Makino et al., 2015). Re-analysis using a large study of 100 Swedish infants and a shotgun metagenomics approach (Bäckhed et al., 2015; Nayfach et al., 2016), may help to explain this counterintuitive result. Nyfach and colleagues (2016), limiting the analysis to vaginally delivered infants, showed the similarity of microbial community within mother-infant pairs was no greater than for unrelated pairs. However, a novel approach using rare single nucleotide polymorphisms to track strains revealed that maternal gut strains were indeed found in their infants. These apparently contradictory results are likely due to the fact that community similarity analysis is based on OTU level. OTUs consist of sequences belonging to one bacterial species but many consist of sequence reads from many different strains. The resolution needed to detect mother to infant transfer of specific strains is therefore lacking. Most detected species are present in a majority of infants and mothers and no particular similarity within mother-infant pairs is detectable. When strains uniquely present in the individuals can be detected, the presence of an identical strain in mother and infant strongly corroborates the hypothesis of some maternal to infant transfer. Definitive tracking of microbial transfer requires strain-level analysis, and community similarity analyses can only provide an overall assessment and exclude transfer when the communities are highly dissimilar, such as was the case in vaginal maternal and infant stool microbiota. In our study, it is likely that employing strain-level analysis would reveal strains shared between maternal rectal and infant microbiota related pairs. However, this would account for a small proportion of the infant gut microbiome because a substantial proportion of infant stool microbiota was divergent from the maternal microbiota in community membership so could not have shared the same strains. This conclusion is unaffected by the limited resolution of the analysis based on sequencing of $16 \mathrm{~S}$ rRNA gene.

A strong impact of delivery mode on infant microbiota composition in the first weeks of life is consistent with large number of previous studies. However, there was little evidence that differential transfer of maternal microbiota had a substantial influence on infant microbiota composition. This seems like a paradox. One possible explanation is that the labour process and vaginal birth may impact infant physiology leading to the establishment of specific microbiota in the gut. There is emerging evidence that lack of vaginal delivery, and in particular of labour, may interfere with the maturation of immune cells and may change the levels of circulating cytokines (Almanzar et al., 2015; Thysen et al., 2015). Delivery mode has differential consequences for activation of the foetal hypothalamic-pituitary-adrenal axis, with profound effects on the developing foetal immune system (Yektaei-Karin et al., 2007). Small differences in the perinatal environment can result in considerable differences in gut microbiota of the neonate. For example, it has been observed that among infants born at term, those with shorter gestational age showed slower maturation of microbiota during early infancy, independently of the delivery mode (Dogra et al., 2015). Caesarean section often results from underlying problems, which might contribute to the alternation in microbiota of Caesarean-section delivered infants and elective and emergency Caesarean section lead to distinct patterns of microbiota dysbiosis (Stokholm et al., 2016).

We have described the earliest stage of acquisition of nasal microbiota, and to our knowledge, this study is the first to investigate nasal microbiota very early in life with comprehensive microbiota profiling. The considerable change in infant nasal microbiota from birth to week 3 resulted in marked resemblance to adult nasal microbiota, i.e. domination by Staphylococcus and Corynebacterium as observed previously (Bassis et al., 2014). This is not surprising as the infant and adult nasal mucosae provide a relatively similar environment to bacteria in contrast to the intestine, where breast milk or formula provide specific substrates for foetal gut microbiota contrasting to the niche offered to the adult gut microbiota. One way in which the infant nasal microbiota differed from that of adults' is the $69 \%$ prevalence of colonisation with potentially pathogenic S. aureus, compared with the approximately $30 \%$ prevalence usually found in adults (Wertheim et al., 2005). Our findings are consistent with those of (Mika et al., 2015) where the prevalence of Staphylococceace was highest at the beginning of the observation period in 6 weeks old infants, and then gradually decreased. We found a surprisingly similar microbiota in infant nose to this reported recently for infant nasopharynx (Bosch et al., 2016), a body site which is close but clearly differentiated in adults, suggesting much less differentiation between the two sites in infants.

A limitation of our study is the unavailability of samples in our cohort that would allow investigation of the gut and nasal microbiota of infants delivered by Caesarean section and breastfed, or those exclusively formula fed in combination with either delivery mode. Breast milk samples might have helped to better understand the exact 
transmission route of microbes, but no such samples were available. A further limitation is that because we applied stringent quality controls, our sample size was reduced by inability to generate sequencing data from approximately a third of samples, most likely due to low bacterial load. Our qPCR analysis of total bacterial load corroborated previous results showing that the PCR-based profiling of microbiota is reliable with bacterial loads above $10^{5}$ to $10^{6}$ of bacterial genomes per ml (Biesbroek et al., 2012; Salter et al., 2014). It has been suggested that many microbiota studies generate erroneous results when the sequencing approach developed for bacteria-rich samples, such as gut or vagina, are applied without modification to bacteria-poor sites (Lauder et al., 2016; Salter et al., 2014; Yong, 2014). We have not investigated maternal nasal microbiota, which is known to be a frequent source of $S$. aureus for infants (Peacock et al., 2003). However, the absence of $S$. aureus from maternal rectum, vagina and breast skin suggest that transmission from mother to infant would occur directly from the mother's nose and not from other body sites. An additional limitation is that we used maternal rectal swabs to sample the maternal gastrointestinal tract as stool samples were not available. We were able to detect OTUs unique to maternal rectum compared to other maternal sites. Many represented strict anaerobes known to comprise the adult human gut microbiome. However, we expect that the proportions of taxa in the rectal swabs are different to that which would be found in the GI tract and so used Binary Jaccard community similarity metrics which take into account only presence or absence data. Finally, analysis of 16S data using the QIME and MOTHUR pipelines is known to result in calling of spurious OTUs. To minimise these problems we eliminated samples with low DNA content and indeed we detected only 436 OTUs. However, even a sizeable fraction of spurious OTUs would not change the conclusion that there is minimal overlap between maternal vaginal and infant stool microbiota.

In conclusion, we have observed substantial effects of delivery mode and feeding mode on the gut microbiota of the infants accompanied by very limited similarity between infant and own mother's vaginal microbiota, for most infants. This suggests that the impact of Caesarean section on infant gut microbiota composition is not wholly explained by reduced transfer of maternal vaginal microbes. The effect of delivery mode may rather be linked to physiological conditions such as differences in immune maturation and/or differences in the transient priming of the microbiota. Further studies (such as investigation of differential cytokine and hormone levels in cord blood from infants born by different delivery modes) are warranted to disentangle immunological from microbial imprinting. It will be important to explain why a small proportion of infants share the majority of their faecal microbiota members with their mothers but most do not, as well as to investigate other potential sources of early microbial gut colonisers. For instance breast milk may contain viable microbes (albeit in small numbers) at the same time exerting potent prebiotic effect (McGuire et al., 2016). The interplay of altered gut microbiota composition and immune maturation should be further explored in the context of increased prevalence of chronic immune disorders seen in Caesarean section delivered infants (Sevelsted et al., 2015).

\section{Supplementary material}

Supplementary material can be found online at https://doi. org/10.3920/BM2017.0064.

Figure S1. Alpha diversity in different sample types.

Figure S2. Bray Curtis within-niche distances in different sample types.

Figure S3. Enumeration of total lactobacilli by qPCR.

Table S1. List of operational taxonomic units identified in maternal rectal samples.

Table S2. Number of samples which provided sequencing data.

Table S3. Pairwise comparison of community distances between sample types.

Table S4. Characteristics of mothers and infants which were included in microbiota analysis by birth and feeding mode.

Table S5. The dynamics of community similarities (Bray Curtis distances) between maternal samples collected at birth and infant samples.

Table S6. Binary Jaccard distances between microbiota of infant stool at day 3 to maternal microbiotas.

Table S7. Binary Jaccard distances between microbiota of infant stool at week 3 to maternal microbiotas.

Table S8. Binary Jaccard distances between infant nasal microbiota at week 3 to maternal microbiotas.

Table S9. Binary Jaccard distances between microbiota of infant stool at day 3 to maternal microbiotas.

Table S10. Binary Jaccard distances between microbiota of infant stool at week 3 to maternal microbiotas.

Table S11. Binary Jaccard distances between infant nasal microbiota at birth to maternal microbiotas.

Table S12. Binary Jaccard distances between infant nasal microbiota at week 3 to maternal microbiotas. 


\section{Conflict of interest}

We would like to disclose that OS, FF, SC, BB and $\mathrm{HB}$ are employees of the Nestlé Research Center. Nestec provided support in the form of salaries for authors but it did not have any additional role in the study design, data collection and analysis, decision to publish, or preparation of the manuscript. KMG, CYS and MJM have received reimbursement for speaking at conferences sponsored by companies selling nutritional products, and are part of an academic consortium that has received research funding from Abbott Nutrition, Nestec and Danone. The other authors have no conflicts of interest.

\section{Acknowledgements}

We thank Irma Silva Zolezzi, Nabil Bosco, Laurent Favre, Jose (Pepe) Saavedra for helpful comments and discussions and Wolfram Brück for the help with the organization of analyses. We would also like to thank Anne Cecile Pittet and Nadine Porta for technical support.

The GUSTO study group includes Allan Sheppard, Amutha Chinnadurai, Anne Eng Neo Goh, Anne Rifkin-Graboi, Anqi Qiu, Arijit Biswas, Bee Wah Lee, Birit F.P. Broekman, Boon Long Quah, Borys Shuter, Carolina Un Lam, Chai Kiat Chng, Cheryl Ngo, Choon Looi Bong, Christiani Jeyakumar Henry, Claudia Chi, Cornelia Yin Ing Chee, Yam Thiam Daniel Goh, Doris Fok, E Shyong Tai, Elaine Tham, Elaine Quah Phaik Ling, Evelyn Xiu Ling Loo, Fabian Yap, Falk Mueller-Riemenschneider, George Seow Heong Yeo, Helen Chen, Heng Hao Tan, Hugo P S van Bever, Iliana Magiati, Inez Bik Yun Wong, Ivy Yee-Man Lau, Izzuddin Bin Mohd Aris, Jeevesh Kapur, Jenny L. Richmond, Jerry Kok Yen Chan, Joanna D. Holbrook, Joanne Yoong, Joao N. Ferreira., Jonathan Tze Liang Choo, Jonathan Y. Bernard, Joshua J. Gooley, Keith M. Godfrey, Kenneth Kwek, Kok Hian Tan, Krishnamoorthy Niduvaje, Kuan Jin Lee, Leher Singh, Lieng Hsi Ling, Lin Lin Su, Ling-Wei Chen, Lourdes Mary Daniel, Lynette Pei-Chi Shek, Marielle V. Fortier, Mark Hanson, Mary Foong-Fong Chong, Mary Rauff, Mei Chien Chua, Melvin Khee-Shing Leow, Michael Meaney, Mya Thway Tint, Neerja Karnani, Ngee Lek, Oon Hoe Teoh, P. C. Wong, Paulin Tay Straughan, Peter D. Gluckman, Pratibha Agarwal, Queenie Ling Jun Li, Rob M. van Dam, Salome A. Rebello, Seang-Mei Saw, See Ling Loy, S. Sendhil Velan, Seng Bin Ang, Shang Chee Chong, Sharon Ng, Shiao-Yng Chan, Shirong Cai, Shu-E Soh, Sok Bee Lim, Stella Tsotsi, Chin-Ying Stephen Hsu, Sue Anne Toh, Swee Chye Quek, Victor Samuel Rajadurai, Walter Stunkel, Wayne Cutfield, Wee Meng Han, Wei Wei Pang, Yap-Seng Chong, Yin Bun Cheung, Yiong Huak Chan, Yung Seng Lee and Zhongwei Huang.

\section{References}

Adlerberth, I. and Wold, A.E., 2009. Establishment of the gut microbiota in Western infants. Acta Paediatrica 98: 229-238.

Alarcón, B., Vicedo, B. and Aznar, R., 2006. PCR-based procedures for detection and quantification of Staphylococcus aureus and their application in food. Journal of Applied Microbiology 100: 352-364.

Almanzar, G., Schönlaub, J., Hammerer-Lercher, A., Koppelstaetter, C., Bernhard, D. and Prelog, M., 2015. Influence of the delivery modus on subpopulations and replication of lymphocytes in mothers and newborns. Early Human Development 91: 663-670.

Arrieta, M.C., Stiemsma, L.T., Dimitriu, P.A., Thorson, L., Russell, S., Yurist-Doutsch, S., Kuzeljevic, B., Gold, M.J., Britton, H.M., Lefebvre, D.L., Subbarao, P., Mandhane, P., Becker, A., McNagny, K.M., Sears, M.R., Kollmann, T., Mohn, W.W., Turvey, S.E. and Finlay, B.B., 2015. Early infancy microbial and metabolic alterations affect risk of childhood asthma. Science Translational Medicine 7: 307ra152.

Azad, M.B., Konya, T., Guttman, D.S., Field, C.J., Sears, M.R., HayGlass, K.T., Mandhane, P.J., Turvey, S.E., Subbarao, P., Becker, A.B., Scott, J.A., Kozyrskyj, A.L. and the CHILD study investigators, 2015. Infant gut microbiota and food sensitization: associations in the first year of life. Clinical and Experimental Allergy 45: 632-643.

Azad, M.B., Konya, T., Maughan, H., Guttman, D.S., Field, C.J., Chari, R.S., Sears, M.R., Becker, A.B., Scott, J.A. and Kozyrskyj, A.L., 2013. Gut microbiota of healthy Canadian infants: profiles by mode of delivery and infant diet at 4 months. Canadian Medical Association Journal 185: 385-394.

Bäckhed, F., Roswall, J., Peng, Y., Feng, Q., Jia, H., Kovatcheva-Datchary, P., Li, Y., Xia, Y., Xie, H., Zhong, H., Khan, M.T., Zhang, J., Li, J., Xiao, L., Al-Aama, J., Zhang, D., Lee, Y.S., Kotowska, D., Colding, C., Tremaroli, V., Yin, Y., Bergman, S., Xu, X., Madsen, L., Kristiansen, K., Dahlgren, J. and Jun, W., 2015. Dynamics and stabilization of the human gut microbiome during the first year of life. Cell Host and Microbe 17: 690-703.

Bassis, C.M., Tang, A.L., Young, V.B. and Pynnonen, M.A., 2014. The nasal cavity microbiota of healthy adults. Microbiome 2: 27.

Biesbroek, G., Sanders, E.A.M., Roeselers, G., Wang, X., Caspers, M.P.M., Trzciński, K., Bogaert, D. and Keijser, B.J.F., 2012. Deep sequencing analyses of low density microbial communities: working at the boundary of accurate microbiota detection. PLoS ONE 7: e32942.

Bisgaard, H., Hermansen, M.N., Buchvald, F., Loland, L., Halkjaer, L.B., Bonnelykke, K., Brasholt, M., Heltberg, A., Vissing, N.H., Thorsen, S.V., Stage, M. and Pipper, C.B., 2007. Childhood asthma after bacterial colonization of the airway in neonates. New England Journal of Medicine 357: 1487-1495.

Bisgaard, H., Li, N., Bonnelykke, K., Chawes, B.L.K., Skov, T., PaludanMüller, G., Stokholm, J., Smith, B. and Krogfelt, K.A., 2011. Reduced diversity of the intestinal microbiota during infancy is associated with increased risk of allergic disease at school age. Journal of Allergy and Clinical Immunology 128: 646-652. 
Bokulich, N.A., Chung, J., Battaglia, T., Henderson, N., Jay, M., Li, H., Lieber, D.A., Wu, F., Perez-Perez, G.I., Chen, Y., Schweizer, W., Zheng, X., Contreras, M., Dominguez-Bello, M.G. and Blaser, M.J., 2016. Antibiotics, birth mode, and diet shape microbiome maturation during early life. Science Translational Medicine 8: 343 ra382.

Bokulich, N.A., Subramanian, S., Faith, J.J., Gevers, D., Gordon, J.I., Knight, R., Mills, D.A. and Caporaso, J.G., 2013. Quality-filtering vastly improves diversity estimates from Illumina amplicon sequencing. Nature Methods 10: 57-59.

Bosch, A.A.T.M., Levin, E., Van Houten, M.A., Hasrat, R., Kalkman, G., Biesbroek, G., De Steenhuijsen Piters, W.A.A., De Groot, P.K.C.M., Pernet, P., Keijser, B.J.F., Sanders, E.A.M. and Bogaert, D., 2016. Development of upper respiratory tract microbiota in infancy is affected by mode of delivery. EBioMedicine 9: 336-345.

Caporaso, J.G., Kuczynski, J., Stombaugh, J., Bittinger, K., Bushman, F.D., Costello, E.K., Fierer, N., Pẽa, A.G., Goodrich, J.K., Gordon, J.I., Huttley, G.A., Kelley, S.T., Knights, D., Koenig, J.E., Ley, R.E., Lozupone, C.A., McDonald, D., Muegge, B.D., Pirrung, M., Reeder, J., Sevinsky, J.R., Turnbaugh, P.J., Walters, W.A., Widmann, J., Yatsunenko, T., Zaneveld, J. and Knight, R., 2010. QIIME allows analysis of high-throughput community sequencing data. Nature Methods 7: 335-336.

Chu, D.M., Ma, J., Prince, A.L., Antony, K.M., Seferovic, M.D. and Aagaard, K.M., 2017. Maturation of the infant microbiome community structure and function across multiple body sites and in relation to mode of delivery. Nature Medicine 23: 314-326.

David, L.A., Weil, A., Ryan, E.T., Calderwood, S.B., Harris, J.B., Chowdhury, F., Begum, Y., Qadri, F., LaRocque, R.C. and Turnbaugh, P.J., 2015. Gut microbial succession follows acute secretory diarrhea in humans. mBio 6: 1-14.

Dogra, S., Sakwinska, O., Soh, S.-E., Ngom-Bru, C., Brück, W.M., Berger, B., Brüssow, H., Lee, Y.S., Yap, F., Chong, Y.-S., Godfrey, K.M. and Holbrook, J.D., 2015. Dynamics of infant gut microbiota are influenced by delivery mode and gestational duration and are associated with subsequent adiposity. mBio 6: e02419-02414.

Dominguez-Bello, M.G., Costello, E.K., Contreras, M., Magris, M., Hidalgo, G., Fierer, N. and Knight, R., 2010. Delivery mode shapes the acquisition and structure of the initial microbiota across multiple body habitats in newborns. Proceedings of the National Academy of Sciences of the USA 107: 11971-11975.

Dominguez-Bello, M.G., De Jesus-Laboy, K.M., Shen, N., Cox, L.M., Amir, A., Gonzalez, A., Bokulich, N.A., Song, S.J., Hoashi, M., Rivera-Vinas, J.I., Mendez, K., Knight, R. and Clemente, J.C., 2016. Partial restoration of the microbiota of cesarean-born infants via vaginal microbial transfer. Nature Medicine 22: 250-253.

Edgar, R.C., 2010. Search and clustering orders of magnitude faster than BLAST. Bioinformatics 26: 2460-2461.

Edgar, R.C., Haas, B.J., Clemente, J.C., Quince, C. and Knight, R., 2011. UCHIME improves sensitivity and speed of chimera detection. Bioinformatics 27: 2194-2200.

Faust, K. and Raes, J., 2012. Microbial interactions: from networks to models. Nature Reviews Microbiology 10: 538-550.

Gensollen, T., Iyer, S.S., Kasper, D.L. and Blumberg, R.S., 2016. How colonization by microbiota in early life shapes the immune system. Science 352: 539-544.
Huttenhower, C., Gevers, D., Knight, R., Abubucker, S., Badger, J.H., Chinwalla, A.T., Creasy, H.H., Earl, A.M., Fitzgerald, M.G., Fulton, R.S., Giglio, M.G., Hallsworth-Pepin, K., Lobos, E.A., Madupu, R., Magrini, V., Martin, J.C., Mitreva, M., Muzny, D.M., Sodergren, E.J., Versalovic, J., Wollam, A.M., Worley, K.C., Wortman, J.R., Young, S.K., Zeng, Q., Aagaard, K.M., Abolude, O.O., Allen-Vercoe, E., Alm, E.J., Alvarado, L., Andersen, G.L., Anderson, S., Appelbaum, E., Arachchi, H.M., Armitage, G., Arze, C.A., Ayvaz, T., Baker, C.C., Begg, L., Belachew, T., Bhonagiri, V., Bihan, M., Blaser, M.J., Bloom, T., Bonazzi, V., Paul Brooks, J., Buck, G.A., Buhay, C.J., Busam, D.A., Campbell, J.L., Canon, S.R., Cantarel, B.L., Chain, P.S.G., Chen, I.M.A., Chen, L., Chhibba, S., Chu, K., Ciulla, D.M., Clemente, J.C., Clifton, S.W., Conlan, S., Crabtree, J., Cutting, M.A., Davidovics, N.J., Davis, C.C., Desantis, T.Z., Deal, C., Delehaunty, K.D., Dewhirst, F.E., Deych, E., Ding, Y., Dooling, D.J., Dugan, S.P., Michael Dunne, W., Scott Durkin, A., Edgar, R.C., Erlich, R.L., Farmer, C.N., Farrell, R.M., Faust, K., Feldgarden, M., Felix, V.M., Fisher, S., Fodor, A.A., Forney, L.J., Foster, L., Di Francesco, V., Friedman, J., Friedrich, D.C., Fronick, C.C., Fulton, L.L., Gao, H., Garcia, N., Giannoukos, G., Giblin, C., Giovanni, M.Y., Goldberg, J.M., Goll, J., Gonzalez, A., Griggs, A., Gujja, S., Kinder Haake, S., Haas, B.J., Hamilton, H.A., Harris, E.L., Hepburn, T.A., Herter, B., Hoffmann, D.E., Holder, M.E., Howarth, C., Huang, K.H., Huse, S.M., Izard, J., Jansson, J.K., Jiang, H., Jordan, C., Joshi, V., Katancik, J.A., Keitel, W.A., Kelley, S.T., Kells, C., King, N.B., Knights, D., Kong, H.H., Koren, O., Koren, S., Kota, K.C., Kovar, C.L., Kyrpides, N.C., La Rosa, P.S., Lee, S.L., Lemon, K.P., Lennon, N., Lewis, C.M., Lewis, L., Ley, R.E., Li, K., Liolios, K., Liu, B., Liu, Y., Lo, C.C., Lozupone, C.A., Dwayne Lunsford, R., Madden, T., Mahurkar, A.A., Mannon, P.J., Mardis, E.R., Markowitz, V.M., Mavromatis, K., McCorrison, J.M., McDonald, D., McEwen, J., McGuire, A.L., McInnes, P., Mehta, T., Mihindukulasuriya, K.A., Miller, J.R., Minx, P.J., Newsham, I., Nusbaum, C., Oglaughlin, M., Orvis, J., Pagani, I., Palaniappan, K., Patel, S.M., Pearson, M., Peterson, J., Podar, M., Pohl, C., Pollard, K.S., Pop, M., Priest, M.E., Proctor, L.M., Qin, X., Raes, J., Ravel, J., Reid, J.G., Rho, M., Rhodes, R., Riehle, K.P., Rivera, M.C., Rodriguez-Mueller, B., Rogers, Y.H., Ross, M.C., Russ, C., Sanka, R.K., Sankar, P., Fah Sathirapongsasuti, J., Schloss, J.A., Schloss, P.D., Schmidt, T.M., Scholz, M., Schriml, L., Schubert, A.M., Segata, N., Segre, J.A., Shannon, W.D., Sharp, R.R., Sharpton, T.J., Shenoy, N., Sheth, N.U., Simone, G.A., Singh, I., Smillie, C.S., Sobel, J.D., Sommer, D.D., Spicer, P., Sutton, G.G., Sykes, S.M., Tabbaa, D.G., Thiagarajan, M., Tomlinson, C.M., Torralba, M., Treangen, T.J., Truty, R.M., Vishnivetskaya, T.A., Walker, J., Wang, L., Wang, Z., Ward, D.V., Warren, W., Watson, M.A., Wellington, C., Wetterstrand, K.A., White, J.R., Wilczek-Boney, K., Wu, Y., Wylie, K.M., Wylie, T., Yandava, C., Ye, L., Ye, Y., Yooseph, S., Youmans, B.P., Zhang, L., Zhou, Y., Zhu, Y., Zoloth, L., Zucker, J.D., Birren, B.W., Gibbs, R.A., Highlander, S.K., Methé, B.A., Nelson, K.E., Petrosino, J.F., Weinstock, G.M., Wilson, R.K. and White, O., 2012. Structure, function and diversity of the healthy human microbiome. Nature 486: 207-214.

Jost, T., Lacroix, C., Braegger, C.P., Rochat, F. and Chassard, C., 2013. Vertical mother-neonate transfer of maternal gut bacteria via breastfeeding. Environmental Microbiology 16: 2891-2904. 
Korpela, K., Salonen, A., Virta, L.J., Kekkonen, R.A. and De Vos, W.M., 2016. Association of early-life antibiotic use and protective effects of breastfeeding: role of the intestinal microbiota. JAMA Pediatrics 170: 750-757.

Lauder, A.P., Roche, A.M., Sherrill-Mix, S., Bailey, A., Laughlin, A.L., Bittinger, K., Leite, R., Elovitz, M.A., Parry, S. and Bushman, F.D., 2016. Comparison of placenta samples with contamination controls does not provide evidence for a distinct placenta microbiota. Microbiome 4: 29.

Madan, J.C., Hoen, A.G., Lundgren, S.N., Farzan, S.F., Cottingham, K.L., Morrison, H.G., Sogin, M.L., Li, H., Moore, J.H., Karagas, M.R., 2016. Association of cesarean delivery and formula supplementation with the intestinal microbiome of 6 -week-old infants. JAMA Pediatrics 170: 212-219.

Makino, H., Martin, R., Ishikawa, E., Gawad, A., Kubota, H., Sakai, T., Oishi, K., Tanaka, R., Ben-Amor, K., Knol, J. and Kushiro, A., 2015. Multilocus sequence typing of bifidobacterial strains from infant's faeces and human milk: are bifidobacteria being sustainably shared during breastfeeding? Beneficial Microbes 6: 563-572.

McDonald, D., Price, M.N., Goodrich, J., Nawrocki, E.P., Desantis, T.Z., Probst, A., Andersen, G.L., Knight, R. and Hugenholtz, P., 2012. An improved Greengenes taxonomy with explicit ranks for ecological and evolutionary analyses of bacteria and archaea. ISME Journal 6: 610-618.

McGuire, M.K., McGuire, M.A. and Bode, L., 2016. Prebiotics and probiotics in human milk: origins and functions of milk-borne oligosaccharides and bacteria. Elsevier, Amsterdam, the Netherlands.

Mika, M., Mack, I., Korten, I., Qi, W., Aebi, S., Frey, U., Latzin, P. and Hilty, M., 2015. Dynamics of the nasal microbiota in infancy: a prospective cohort study. Journal of Allergy and Clinical Immunology 135: 905-912.

Nadkarni, M.A., Martin, F.E., Jacques, N.A. and Hunter, N., 2002. Determination of bacterial load by real-time PCR using a broadrange (universal) probe and primers set. Microbiology 148: 257-266.

Nayfach, S., Rodriguez-Mueller, B., Garud, N. and Pollard, K.S., 2016. An integrated metagenomics pipeline for strain profiling reveals novel patterns of bacterial transmission and biogeography. Genome Research 26: 1612-1625

Peacock, S.J., Justice, A., Griffiths, D., De Silva, G.D.I., Kantzanou, M.N., Crook, D., Sleeman, K. and Day, N.P.J., 2003. Determinants of acquisition and carriage of Staphylococcus aureus in infancy. Journal of Clinical Microbiology 41: 5718-5725.

Penders, J., Thijs, C., Vink, C., Stelma, F.F., Snijders, B., Kummeling, I., Van den Brandt, P.A. and Stobberingh, E.E., 2006. Factors influencing the composition of the intestinal microbiota in early infancy. Pediatrics 118: 511-521.

Prior, E., Santhakumaran, S., Gale, C., Philipps, L.H., Modi, N. and Hyde, M.J., 2012. Breastfeeding after cesarean delivery: a systematic review and meta-analysis of world literature. American Journal of Clinical Nutrition 95: 1113-1135.

Quince, C., Lanzén, A., Curtis, T.P., Davenport, R.J., Hall, N., Head, I.M., Read, L.F. and Sloan, W.T., 2009. Accurate determination of microbial diversity from 454 pyrosequencing data. Nature Methods 6: 639-641.
Salter, S.J., Cox, M.J., Turek, E.M., Calus, S.T., Cookson, W.O., Moffatt, M.F., Turner, P., Parkhill, J., Loman, N.J. and Walker, A.W., 2014. Reagent and laboratory contamination can critically impact sequence-based microbiome analyses. BMC Biology 12: 1-12.

Schloss, P.D., Westcott, S.L., Ryabin, T., Hall, J.R., Hartmann, M., Hollister, E.B., Lesniewski, R.A., Oakley, B.B., Parks, D.H., Robinson, C.J., Sahl, J.W., Stres, B., Thallinger, G.G., Van Horn, D.J. and Weber, C.F., 2009. Introducing mothur: open-source, platform-independent, community-supported software for describing and comparing microbial communities. Applied and Environmental Microbiology 75: 7537-7541.

Sevelsted, A., Stokholm, J., Bønnelykke, K. and Bisgaard, H., 2015. Cesarean section and chronic immune disorders. Pediatrics 135: e92-e98.

Sim, K., Shaw, A.G., Randell, P., Cox, M.J., McClure, Z.E., Li, M.S., Haddad, M., Langford, P.R., Cookson, W.O.C.M., Moffatt, M.F. and Kroll, J.S., 2015. Dysbiosis anticipating necrotizing enterocolitis in very premature infants. Clinical Infectious Diseases 60: 389-397.

Soh, S.E., Saw, S.M., Soh, S.E., Tint, M.T., Chong, Y.S., Gluckman, P.D., Rifkin-Graboi, A., Stünkel, W., Holbrook, J.D., Gluckman, P.D., Godfrey, K.M., Godfrey, K.M., Chan, Y.H., Kwek, K., Sheppard, A., Chinnadurai, A., Ferguson-Smith, A., Biswas, A., Chia, U., Leutscher-Broekman, B., Shuter, B., Cai, S., Ngo, C., Chng, C.K., Chong, S.C., Henry, C.J., Chu, M.C., Chee, C.Y.I., Goh, Y.T.D., Bier, D., Ding, C.M., Fok, D., Finkelstein, E.A., Peng Yap, F.K., Yeo, G.S.H., Meng Han, W., Chen, H., Van Bever, H.P.S., Inskip, H., Magiati, I., Kapur, J., Richmond, J.L., Gooley, J.J., Niduvaje, K., Lee, B.W., Lee, Y.S., Singh, L., Lim, S.B., Daniel, L.M., Loh, S.F., Low, Y.L., Fortier, M., Hanson, M., Chong, M.F.F., Meaney, M., Morton, S., Pang, W.W., Agarwal, P., Qiu, A., Quah, B.L., Van Dam, R.M., Stringer, D., Rebello, S.A., So, W.C., Hsu, C.Y., Su, L.L., Tang, J., Tan, K.H., Tan, S.H., Teoh, O.H., Rajadurai, V.S., Wong, P.C. and Venkatesh, S.K., 2014. Cohort profile: growing up in Singapore towards healthy outcomes (GUSTO) birth cohort study. International Journal of Epidemiology 43: 1401-1409.

Stokholm, J., Thorsen, J., Chawes, B.L., Schjørring, S., Krogfelt, K.A., Bønnelykke, K. and Bisgaard, H., 2016. Cesarean section changes neonatal gut colonization. Journal of Allergy and Clinical Immunology 138: 881-889.

Teo, S.M., Mok, D., Pham, K., Kusel, M., Serralha, M., Troy, N., Holt, B.J., Hales, B.J., Walker, M.L., Hollams, E., Bochkov, Y.A., Grindle, K., Johnston, S.L., Gern, J.E., Sly, P.D., Holt, P.G., Holt, K.E. and Inouye, M., 2015. The infant nasopharyngeal microbiome impacts severity of lower respiratory infection and risk of asthma development. Cell Host and Microbe 17: 704-715.

Thysen, A.H., Larsen, J.M., Rasmussen, M.A., Stokholm, J., Bønnelykke, K., Bisgaard, H. and Brix, S., 2015. Prelabor cesarean section bypasses natural immune cell maturation. Journal of Allergy and Clinical Immunology 136: 1123-1125.

Van den Bergh, M.R., Biesbroek, G., Rossen, J.W.A., De Steenhuijsen Piters, W.A.A., Bosch, A.A.T.M., Van Gils, E.J.M., Wang, X., Boonacker, C.W.B., Veenhoven, R.H., Bruin, J.P., Bogaert, D. and Sanders, E.A.M., 2012. Associations between pathogens in the upper respiratory tract of young children: interplay between viruses and bacteria. PLoS ONE 7: e47711. 
Wang, Q., Garrity, G.M., Tiedje, J.M. and Cole, J.R., 2007. Naïve Bayesian classifier for rapid assignment of rRNA sequences into the new bacterial taxonomy. Applied and Environmental Microbiology 73: 5261-5267.

Warner, B.B., Deych, E., Zhou, Y., Hall-Moore, C., Weinstock, G.M., Sodergren, E., Shaikh, N., Hoffmann, J.A., Linneman, L.A., Hamvas, A., Khanna, G., Rouggly-Nickless, L.C., Ndao, I.M., Shands, B.A., Escobedo, M., Sullivan, J.E., Radmacher, P.G., Shannon, W.D. and Tarr, P.I., 2016. Gut bacteria dysbiosis and necrotising enterocolitis in very low birthweight infants: a prospective case-control study. The Lancet 387: 1928-1936.

Wertheim, H.F.L., Melles, D.C., Vos, M.C., Van Leeuwen, W., Van Belkum, A., Verbrugh, H.A. and Nouwen, J.L., 2005. The role of nasal carriage in Staphylococcus aureus infections. The Lancet Infectious Diseases 5: 751-762.
Yatsunenko, T., Rey, F.E., Manary, M.J., Trehan, I., Dominguez-Bello, M.G., Contreras, M., Magris, M., Hidalgo, G., Baldassano, R.N., Anokhin, A.P., Heath, A.C., Warner, B., Reeder, J., Kuczynski, J., Caporaso, J.G., Lozupone, C.A., Lauber, C., Clemente, J.C., Knights, D., Knight, R. and Gordon, J.I., 2012. Human gut microbiome viewed across age and geography. Nature 486: 222-227.

Yektaei-Karin, E., Moshfegh, A., Lundahl, J., Berggren, V., Hansson, L.O. and Marchini, G., 2007. The stress of birth enhances in vitro spontaneous and IL-8-induced neutrophil chemotaxis in the human newborn. Pediatric Allergy and Immunology 18: 643-651.

Yong, E., 2014. Contaminomics: why some microbiome studies may be wrong. Not exactly rocket science (blog): 11 November 2014. Available at: http://tinyurl.com/ybwj3o5h. 